\title{
Complete Chloroplast Genome Sequencing and Phylogenetic Analysis of Two Dracocephalum Plants
}

\author{
Junjun Yao, ${ }^{1}$ Fangyu Zhao, ${ }^{1}$ Yuanjiang Xu, ${ }^{1,2}$ Kaihui Zhao, ${ }^{1}$ Hong Quan, ${ }^{1,2}$ Yanjie Su, ${ }^{1}$ \\ Peiyu Hao, ${ }^{1}$ Jiang Liu, ${ }^{3}$ Benxia Yu, ${ }^{3}$ Min Yao, ${ }^{4}$ Xiaojing Ma, ${ }^{5}$ Zhihua Liao, ${ }^{6}$ \\ and Xiaozhong Lan $\mathbb{D}^{1,5,6}$ \\ ${ }^{1}$ TAAHC-SWU Medicinal Plant Joint R\&D Center, Tibetan Collaborative Innovation Center of Agricultural and Animal \\ Husbandry Resources, Food Science College, Tibet Agriculture \& Animal Husbandry University, Nyingchi, Tibet 860000, China \\ ${ }^{2}$ Key Laboratory of Forest Ecology in Tibet Plateau (Tibet Agricultural \& Animal Husbandry University), Ministry of Education, \\ Nyingchi, Tibet 860000, China \\ ${ }^{3}$ Chongqing Academy of Chinese Materia Medica, Chongqing 400065, China \\ ${ }^{4}$ State Key Laboratory of Dao-di Herbs, National Resource Center for Chinese Materia Medica, China Academy of Chinese \\ Medical Sciences, Beijing 100700, China \\ ${ }_{5}^{5}$ Jiangxi Institute for Drug Control, NMPA Key Laboratory of Quality Evaluation of Traditional Chinese Patent Medicine, Nanchang, \\ Jiangxi 330029, China \\ ${ }^{6}$ Key Laboratory of Eco-Environments in the Three Gorges Reservoir Region, Ministry of Education, Chongqing Engineering and \\ Technology Research Center for Sweetpotato, School of Life Sciences, Southwest University, Chongqing 400715, China
}

Correspondence should be addressed to Xiaozhong Lan; lanxiaozhong@163.com

Received 27 June 2020; Revised 26 October 2020; Accepted 18 December 2020; Published 30 December 2020

Academic Editor: Sankar Subramanian

Copyright (C) 2020 Junjun Yao et al. This is an open access article distributed under the Creative Commons Attribution License, which permits unrestricted use, distribution, and reproduction in any medium, provided the original work is properly cited.

Dracocephalum tanguticum and Dracocephalum moldavica are important herbs from Lamiaceae and have great medicinal value. We used the Illumina sequencing technology to sequence the complete chloroplast genome of $D$. tanguticum and $D$. moldavica and then conducted de novo assembly. The two chloroplast genomes have a typical quadripartite structure, with the gene's lengths of $82,221 \mathrm{bp}$ and $81,450 \mathrm{bp}$, large single-copy region's (LSC) lengths of 82,221 bp and 81,450 bp, and small single-copy region's (SSC) lengths of 17,363 bp and 17,066 bp, inverted repeat region's (IR) lengths of 51,370 bp and 51,352 bp, respectively. The GC content of the two chloroplast genomes was $37.80 \%$ and $37.83 \%$, respectively. The chloroplast genomes of the two plants encode 133 and 132 genes, respectively, among which there are 88 and 87 protein-coding genes, respectively, as well as 37 tRNA genes and 8 rRNA genes. Among them, the rps2 gene is unique to D. tanguticum, which is not found in D. moldavica. Through SSR analysis, we also found 6 mutation hotspot regions, which can be used as molecular markers for taxonomic studies. Phylogenetic analysis showed that Dracocephalum was more closely related to Mentha.

\section{Introduction}

Lamiaceae is the sixth largest family of phanerogam. There are more than 7,000 species in about 220 genera and 10 subfamilies in the world. In China, there are over 800 species in 99 genera. This family is mainly distributed in Asia, Africa, and Europe. There are about 10 genera with more than 100 species, including Salvia, Mesosphaerum, and Scutellaria, among which the number of species accounts for about two-thirds of the species in this family. The plants of this family draw people's attention because of their aroma and medicinal value $[1,2]$, and they have great economic value. Among them, there are more than 60 kinds of plants in the Dracocephalum, which are mainly distributed in northern Asia, and a few extend to northern Europe or central Europe.

Chloroplast is a kind of plastid and one of the most important organelles in plants and algae cells. In addition to photosynthesis, chloroplasts also participate in the 
TABLE 1: Chloroplast genome information of six species of Lamiaceae.

\begin{tabular}{|c|c|c|c|c|c|c|}
\hline Genome features & D. tanguticum & D. moldavica & S. miltiorrhiza & P. cablin & S. baicalensis & P. officinalis \\
\hline Genome size (bp) & 150,954 & 149,868 & 151,328 & 152,460 & 151,824 & 152,462 \\
\hline LSC size (bp) & 82,221 & 81,450 & 82,695 & 83,974 & 83,976 & 83,355 \\
\hline SSC size (bp) & 17,363 & 17,066 & 17,555 & 17,652 & 17,338 & 17,969 \\
\hline IR size (bp) & 51,370 & 51,352 & 51,078 & 50,834 & 50,510 & 51,138 \\
\hline Number of genes (unique) & $133(114)$ & $132(113)$ & $131(114)$ & $126(109)$ & $132(114)$ & $132(113)$ \\
\hline Protein genes (unique) & $88(8)$ & $87(8)$ & $86(6)$ & $90(8)$ & $87(7)$ & $94(9)$ \\
\hline tRNA genes (unique) & $37(7)$ & $37(7)$ & $37(7)$ & $28(5)$ & $37(7)$ & $30(6)$ \\
\hline rRNA genes (unique) & $8(4)$ & $8(4)$ & $8(4)$ & $8(4)$ & $8(4)$ & $8(4)$ \\
\hline Duplicated genes in IR & 19 & 19 & 17 & 17 & 18 & 19 \\
\hline GC content (\%) & $37.80 \%$ & $37.83 \%$ & $38.00 \%$ & $38.24 \%$ & $38.30 \%$ & $38.00 \%$ \\
\hline GC content in LSC (\%) & $35.84 \%$ & $35.81 \%$ & $35.90 \%$ & $36.30 \%$ & $36.30 \%$ & $36.20 \%$ \\
\hline GC content in SSC (\%) & $31.85 \%$ & $31.79 \%$ & $32.00 \%$ & $32.10 \%$ & $32.70 \%$ & $31.90 \%$ \\
\hline GC content in IR (\%) & $43.04 \%$ & $43.03 \%$ & $43.10 \%$ & $43.50 \%$ & $43.60 \%$ & $43.00 \%$ \\
\hline Total reads & 21517059 & 19384791 & - & - & - & - \\
\hline Aligned paired-end reads & 1531708 & 2709906 & - & - & - & - \\
\hline Average organelle coverage & 3033 & 5412 & - & - & - & - \\
\hline Average insert size (bp) & $323 \pm 60$ & $331 \pm 63$ & - & - & - & - \\
\hline
\end{tabular}
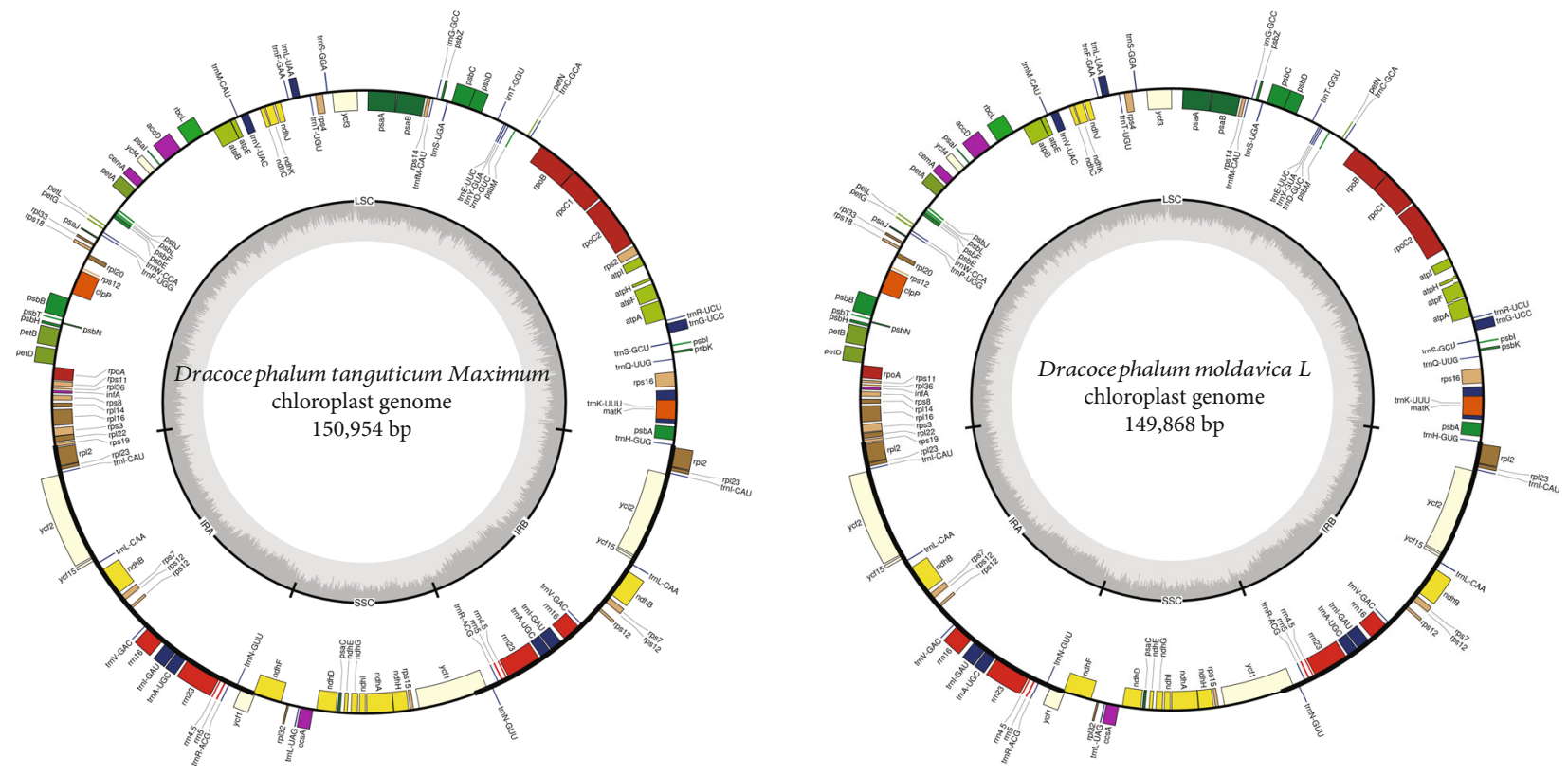

$\begin{array}{ll}\square \text { Photosystem I } & \square \text { Ribosomal proteins (LSU) } \\ \square \text { Photosystem II } & \square \text { clpP, matK } \\ \square \text { Cytochrome b/f complex } & \square \text { Other genes } \\ \square \text { ATP synthase } & \square \text { Hypothetical chloroplast reading frames (ycf) } \\ \square \text { NADH dehydrogenase } & \square \text { ORFs } \\ \square \text { RubisCo large subunit } & \square \text { Transfer RNAs } \\ \square \text { RNA polymerase } & \square \text { Ribosomal RNAs } \\ \square \text { Ribosomal proteins (SSU) } & \square \text { Origin of replication }\end{array}$

(a)

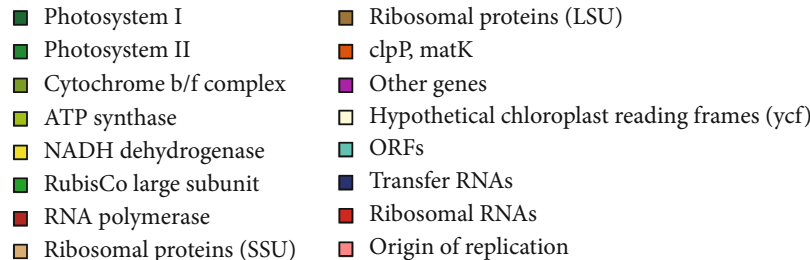

口 Photosystem I

․ Photosystem II

C Cytochrome b/f complex

$\square$ ATP synthase

$\square \mathrm{NADH}$ dehydrogenase

$\square$ RubisCo large subunit

- RNA polymerase

$\square$ Ribosomal proteins (SSU)

(b)

FIGURE 1: (a) D. tanguticum chloroplast genome. (b) D. moldavica chloroplast genome. The genes that code forward are on the outside of the circle, and the genes that code backward are on the inside. The legend identifies genes with different functions. 
TABLe 2: Annotated gene list of chloroplast genomes of D. tanguticum and D. moldavica.

\begin{tabular}{|c|c|c|}
\hline Category of gene & Group of gene & Name of gene \\
\hline \multirow{6}{*}{ Photosynthetic } & Subunits of photosystem I & $p s a A, p s a B, p s a C, p s a I, p s a J$ \\
\hline & Submits of photosystem II & $p s b A, p s b B, p s b C, p s b D, p s b E, p s b F, p s b H, p s b I, p s b J, p s b K, p s b L, p s b M, p s b N, p s b T, p s b Z$ \\
\hline & $\begin{array}{l}\text { Subunits of NADH } \\
\text { dehydrogenase }\end{array}$ & $n d h A, n d h B(x 2), n d h C, n d h D, n d h E, n d h F, n d h G, n d h H, n d h I, n d h J, n d h K$ \\
\hline & $\begin{array}{l}\text { Subunits of cytochrome b/f } \\
\text { complex }\end{array}$ & pet $A, \operatorname{pet} B, \operatorname{pet} D, \operatorname{pet} G, \operatorname{pet} L, \operatorname{pet} N$ \\
\hline & Subunits of ATP synthase & atpA, atpB, atpE, atpF, atpH, atpI \\
\hline & Large subunit of rubisco & $r b c L$ \\
\hline \multirow{5}{*}{ Self-replication } & $\begin{array}{c}\text { Proteins of large ribosomal } \\
\text { subunit }\end{array}$ & rpl2(x2), rpl14, rpl16, rpl20, rpl22, rpl23(x2), rpl32, rpl33, rpl36 \\
\hline & $\begin{array}{c}\text { Proteins of small ribosomal } \\
\text { subunit }\end{array}$ & $r p s 2^{*}, r p s 3, r p s 4, r p s 7(x 2), r p s 8, r p s 11, r p s 12(x 2), r p s 14, r p s 15, r p s 16, r p s 18, r p s 19$ \\
\hline & Subunits of RNA polymerase & rpoA, rрoB, rpoC1, rpoC2 \\
\hline & Ribosomal RNAs & $\operatorname{rrn} 23 S(x 2), \operatorname{rrn} 16 S(x 2), \operatorname{rrn} 5 S(x 2), \operatorname{rrn} 4.5 S(x 2)$ \\
\hline & Transfer RNAs & 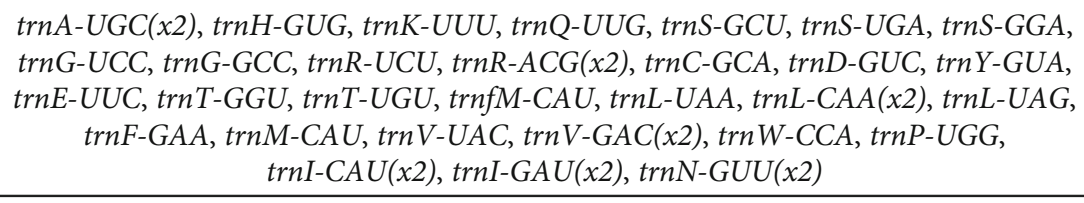 \\
\hline \multirow{6}{*}{ Biosynthesis } & Maturase & $\operatorname{matK}$ \\
\hline & Protease & $c l p P$ \\
\hline & Envelope membrane protein & $\operatorname{cem} A$ \\
\hline & Acetyl-CoA carboxylase & $a c c D$ \\
\hline & $\begin{array}{l}\text { c-type cytochrome synthesis } \\
\text { gene }\end{array}$ & $\operatorname{ccs} A$ \\
\hline & Translation initiation factor & $\operatorname{infA}$ \\
\hline $\begin{array}{l}\text { Unknown } \\
\text { function }\end{array}$ & $\begin{array}{c}\text { Conserved hypothetical } \\
\text { chloroplast } \\
\text { Reading frames }\end{array}$ & $y c f 1(x 2), y c f 2(x 2), y c f 3, y c f 4, y c f 15(x 2)$ \\
\hline
\end{tabular}

${ }^{*}$ Represents the gene unique to $D$. tanguticum.

synthesis of starch, pigments, amino acids, and fatty acids [35]. Chloroplasts are green and oblate under a high-power microscope [6]. The currently accepted view on the origin of chloroplasts is the endosymbiosis theory. The chloroplasts in eukaryotic cells originated from endosymbiont cyanobacteria, which were engulfed and gradually digested by eukaryotic cells before becoming organelles in host cells through a long period of evolution [7]. As semiautonomous genetic organelles, chloroplasts have their own genetic material-chloroplast genome (cpDNA) [8,9]. The length of the genome is about $120-160 \mathrm{~kb}$, and the difference in the size of the chloroplast genome is mainly caused by the shrinkage and expansion of the IR region [10]. For example, the IR size of the chloroplast genome of Pelargonium xhortorum is $75,741 \mathrm{bp}$; the total length of its chloroplast genome is $217,942 \mathrm{bp}$ [11]; the IR of Pinus thunbergii is only $495 \mathrm{bp}$, and its chloroplast genome is only $119,707 \mathrm{bp}$ [12]; the chloroplast genome of Metasequoia glyptostroboides is much shorter due to loss of an IR region [13].

Genome sequencing is often used in the analysis of phylogenetic relationship and research on genetic diversity and evolution $[14,15]$. The three independent genomes that can provide genetic information are chloroplasts, mitochondria, and cell nucleus. Compared with the complexity of the nuclear genome, the nonconservative nature of the large mitochondrial genome, the chloroplast genome is proper in size and easy for sequencing and acquisition [16-18]. Since 1986, the entire chloroplast genome of tobacco was first sequenced successfully [19]. To date, the NCBI GenBank database has collected the complete sequences of chloroplast genomes of more than 1,000 plants. The Lamiaceae family comprises about 200 species. In 2003, Paul Hebert introduced the concept of DNA barcoding, and gene sequencing technology began to be applied to biological species identification systems. Common DNA barcodes used by plants include $r b c L, m a t K, p s b K-p s b I$, trnH-psbA, and ITS [20]. However, using only a single gene for species identification may lead to different results from different genes, which brings about difficulties for species identification [21, 22]. Chloroplast genomes offer more variation than a single gene and will be more effective in addressing the evolutionary rules of close species.

In this study, we used Illumina sequencing technology to conduct full genome sequencing to the chloroplast genome of 
TABLE 3: Intron information of D. tanguticum and D. moldavica.

\begin{tabular}{|c|c|c|c|c|c|c|}
\hline Gene & Location & Exon I (bp) & Intron I (bp) & Exon II (bp) & Intron II (bp) & Exon III (bp) \\
\hline $\operatorname{trnK} K-U U U$ & LSC & 37 & 2544 & 35 & & \\
\hline rps16 & LSC & 42 & 869 & 225 & & \\
\hline $\operatorname{trn} G-U C C$ & LSC & 23 & 682 & 47 & & \\
\hline $\operatorname{atpF}$ & LSC & 152 & 706 & 385 & & \\
\hline rpoC1 & LSC & 456 & 766 & 1620 & & \\
\hline $\operatorname{trnL} L-U A A$ & LSC & 38 & 498 & 49 & & \\
\hline $\operatorname{trn} V-U A C$ & LSC & 35 & 575 & 38 & & \\
\hline rps12 & LSC & 114 & 536 & 258 & & \\
\hline petB & LSC & 7 & 724 & 641 & & \\
\hline petD & LSC & 8 & 708 & 475 & & \\
\hline rpl16 & LSC & 9 & 874 & 399 & & \\
\hline$r p l 2$ & IR & 391 & 661 & 434 & & \\
\hline$n d h B$ & IR & 777 & 677 & 756 & & \\
\hline $\operatorname{trnI}-G A U$ & IR & 37 & 947 & 35 & & \\
\hline $\operatorname{trn} A-U G C$ & IR & 38 & 801 & 35 & & \\
\hline$n d h A$ & SSC & 553 & 1004 & 539 & & \\
\hline$c l p P$ & LSC & 71 & 691 & 292 & 646 & 228 \\
\hline$y c f 3$ & LSC & 129 & 705 & 228 & 746 & 153 \\
\hline
\end{tabular}

D. tanguticum and D. moldavica and then conducted de novo assembly and analysis. Moreover, based on the chloroplast genome data of $D$. tanguticum and D. moldavica and the chloroplast genomes of 30 other species, a phylogenetic tree was constructed. These data will help us better understand the evolutionary history of the branch of Lamiaceae and promote the research on the phylogeny, population, and genetic engineering of medicinal plants.

\section{Results and Discussion}

2.1. Characterization of Chloroplast Genomes in Labiatae Species. A total of $12.27 \mathrm{~Gb}$ raw data were obtained after sequencing. The chloroplast genome size, reads, and GC content of the two Dracocephalum plants are shown in Table 1. The chloroplast genome size of Lamiaceae plants is basically similar, which is about $150 \mathrm{~kb}$. Through genome sequencing and de novo assembly, we obtained the complete chloroplast genome map of D. tanguticum and D. moldavica (Figure 1).

The total length of the chloroplast genomes of $D$. tanguticum and D. moldavica was $150,954 \mathrm{bp}$ and $149,868 \mathrm{bp}$, respectively. Both $D$. tanguticum and $D$. moldavica contain a long single-copy region (LSC) with the lengths of $82,221 \mathrm{bp}$ and $81,450 \mathrm{bp}$ and a short single-copy region (SSC) with the lengths of $17,363 \mathrm{bp}$ and $17,066 \mathrm{bp}$, respectively, which are separated by a pair of inverted repeat regions (IR) with the lengths of $51,370 \mathrm{bp}$ and $51,352 \mathrm{bp}$, respectively (Table 1, Figure 1). The GC content of the chloroplast genome of these Lamiaceae plants is about 38\%, and the GC content of the IR region, LSC region, and SSC region is about $43 \%, 36 \%$, and $32 \%$, respectively. D. tanguticum and D. moldavica encode 133 (114 unique genes) and 132 (113 unique genes) genes, respectively, including 37 tRNA genes and 8 rRNA genes, as well as 88 and 87 protein-coding genes, respectively. In addition, the rps 2 is a gene unique to $D$. tanguticum, which is not found in D. moldavica (Table 2). Gene deletions in other Lamiaceae species have also been reported before, and the deletion of the trnK-UUU gene in the chloroplast genome of Pogostemon cablin has ever been reported [23]. Generally speaking, the IR region is the most conservative region in the chloroplast genome [24]. The expansion and shrinkage of the IR, LSC, and SSC regions during evolution are the main reason for the difference in the length of the chloroplast genome $[15,25]$.

In addition, 18 genes were found to contain intron sequences, of which 16 genes contain one intron and 2 genes contain two introns. Among them, the intron in trnK-UUU is the largest and contains the matK gene (Table 3 ). The size of introns and gene spacers is also a factor that affects the size of the chloroplast genome, and intron loss has been reported in Hordeum vulgare [26, 27]. The two pseudogenes, $y c f 1$ and rps19, are located between IR and SSC and between IRa and LSC, respectively, which is consistent with the research in Prunella vulgaris [28]. CodonW software was used to calculate and analyze the codon usage bias (RSCU) of the chloroplast genome. The protein-coding sequences of the chloroplast genomes of $D$. tanguticum and $D$. moldavica consist of 26,754 and 26,460 codons, respectively. Among them, leucine (Leu) is used the most, and cysteine (Cys) is used the least. Such bias is considered to be a comprehensive result of natural selection, species mutation, and genetic drift $[29,30]$. The complete chloroplast genome with gene annotations has been uploaded to the NCBI database, the accession numbers of D. tanguticum and D. moldavica are MT457746 and MT457747, respectively.

2.2. Comparative Analysis of Genome Structure. The shrinkage and expansion of the boundary region between IR, LSC, 


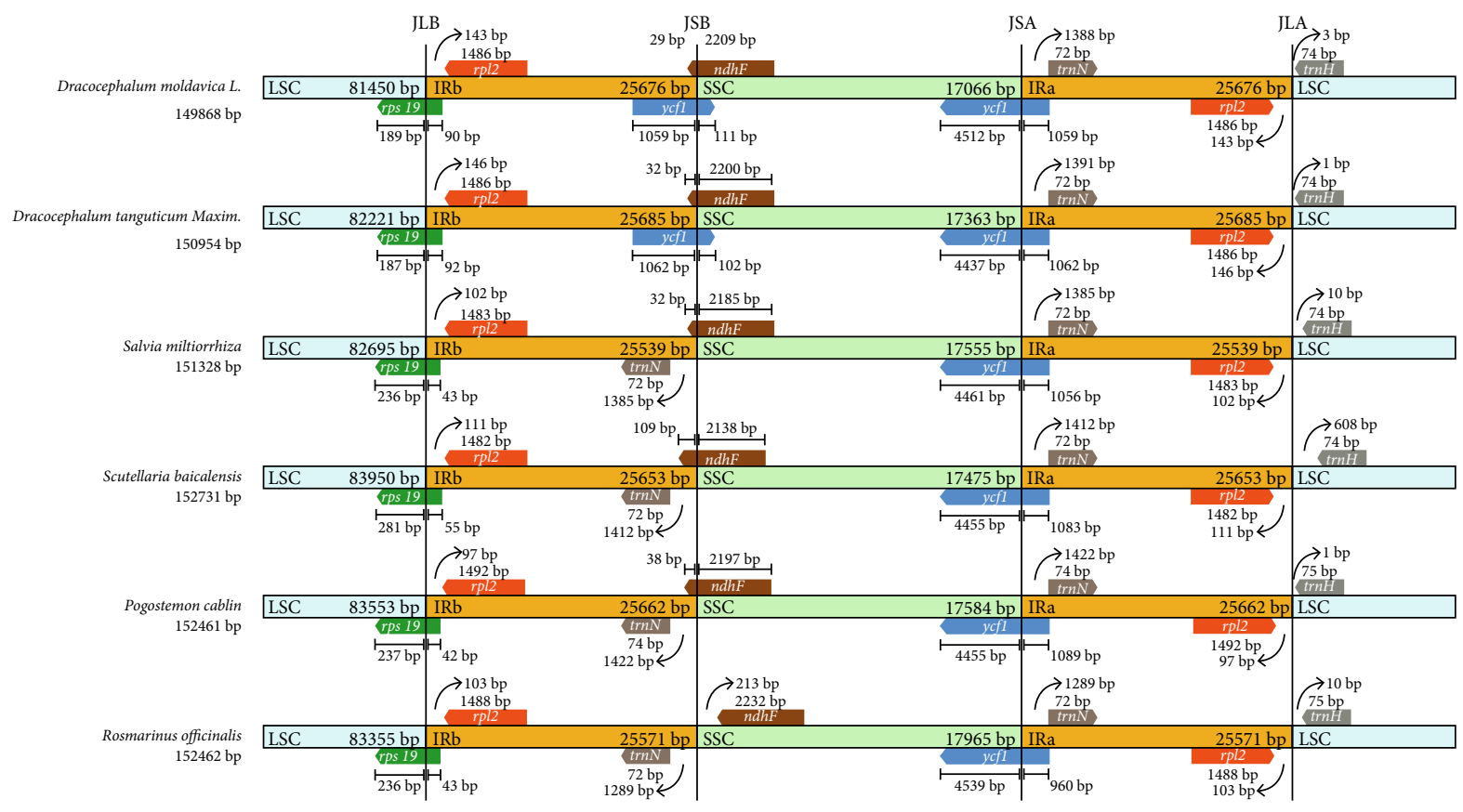

FIgURE 2: Comparison of the borders of LSC, SSC, and IR regions of chloroplast genomes in six Lamiaceae species.

and SSC are an important aspect of the chloroplast genome and are considered to be the main reason for the various sizes of the chloroplast genome $[31,32]$. We analyzed the IR regions of D. moldavica, D. tanguticum, S. miltiorrhiza, $S$. baicalensis, P. cablin, and R. officinalis. The IR scopes of the six Lamiaceae plants are shown in Figure 2.

The rps19 gene is located at the boundary of LSC/IRa. The size of the gene fragment in the LSC region is 187$281 \mathrm{bp}$, and the size of the gene fragment in the IRa region is $42-92 \mathrm{bp}$. The rps 19 and $y c f 1$ genes are usually crossborder genes, which is consistent with the reports on Pongamia pinnata [33]. The rpl2 gene is located at the IR/LSC border, which is $1486 \mathrm{bp}$ in both $D$. tanguticum and $D$. moldavica, but the distance from rpl2 to the border in the D. tanguticum is $2 \mathrm{bp}$ longer than that in the D. moldavica. Additionally, $n d h F$ is also a gene that crosses the IRb/SSC border, but it was found in the analysis of the six Lamiaceae species that the $n d h F$ gene in Rosmarinus officinalis is located in the SSC region, 213 bp away from the border (Figure 2), which is different from that of the other five Lamiaceae plants. Studies have shown that the small amplification of the IR region border is considered to be an important reason for maintaining the stability of the IR region [34]. Goulding et al. proposed the hypothesis of the evolution of the chloroplast's IR region: there is a boundary gene amplification mechanism in the IR region [35].

2.3. Analysis on Simple Repeat Sequences and Mutation Hotspots. We used vmatch v2.3.0 (http://www.vmatch.de/) software to identify the dispersed duplication sequences (Table 4). There were 36, 35 dispersed duplication sequences of $30-60 \mathrm{bp}$ identified in D. tanguticum and D. moldavica, among which there are 18 forward repeats and 18, 17 palindrome repeats, respectively. No reverse and complementary repeat was found. Most of these repeats are between 30 and $40 \mathrm{bp}$. However, the dispersed duplication sequences of $D$.
TABle 4: D. tanguticum and D. moldavica dispersed duplication sequence information.

\begin{tabular}{lcccccc}
\hline $\begin{array}{l}\text { Total } \\
\text { numbers }\end{array}$ & Forward & Palindromic & $\begin{array}{c}\text { Total } \\
\text { regions }\end{array}$ & IGS & Introns & $\begin{array}{c}\text { Coding } \\
\text { region }\end{array}$ \\
\hline 36 & 18 & 18 & 18 & 9 & 2 & 7 \\
35 & 18 & 17 & 17 & 6 & 3 & 8 \\
\hline
\end{tabular}

tanguticum and D. moldavica show great differences. For dispersed duplications between 40 and $60 \mathrm{bp}$, the forward and palindrome repetitions of $D$. tanguticum are evenly distributed, while the palindrome repetitions of $D$. moldavica are significantly more than the forward repetitions.

The mauve software was used to compare the complete chloroplast genome of the two sequenced species with ten other Lamiaceae plants. Comparing the LCBs (local-collinear blocks) of these species, it was found that most of the genes maintained a consistent position and orientation, and the complete genome of the plastid showed a high degree of consistency, with no gene reversal detected. Through comparison of the complete chloroplast genome of 12 Lamiaceae plants, it was found that the rpl2 gene was in the first location in the LSC region in Lavandula angustifolia, while the other 11 plants were in the last location in the IR region (Figure 3). This is different from predecessors' studies [36, 37]. The two differences can be used as the basis for species identification and molecular markers.

With MISA v1.0 (http://pgrc.ipk-gatersleben.de/misa/ misa.html) software, we found 51 and 54 SSRs with at least $10 \mathrm{bp}$ in $D$. tanguticum and D. moldavica, respectively (Table 5). Single-, double-, three-, and four-nucleotide SSRs were detected in D. tanguticum and D. moldavica, and one five-nucleotide SSR was also detected in D. moldavica. However, among them, single nucleotides in D. tanguticum and 


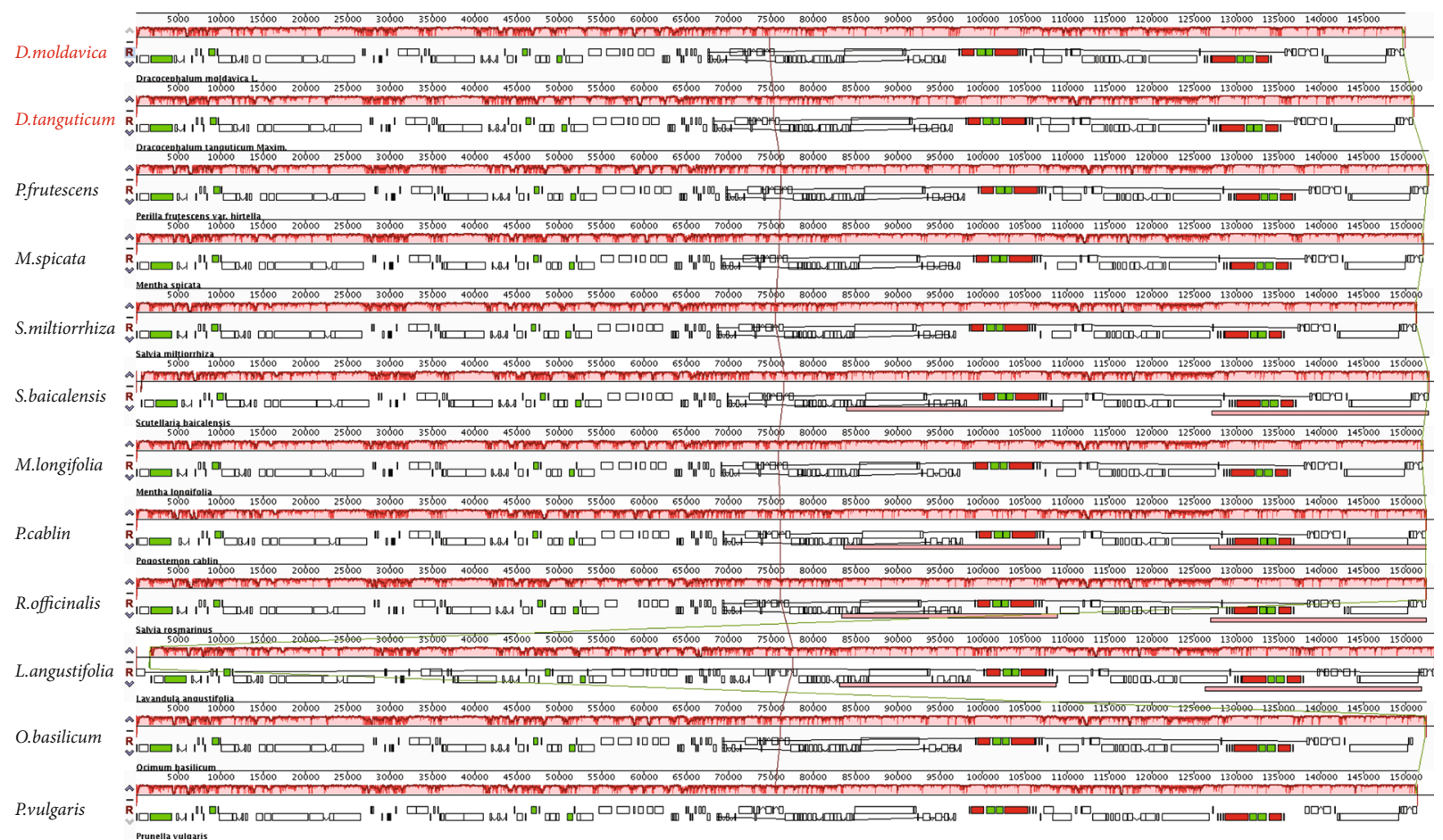

FIGURE 3: Chloroplast gene sequence alignment of 12 species of Lamiaceae. The rectangles represent the locations of genes in each genome. White represents CDs, green represents tRNA, and red represents rRNA.

TABLe 5: D. tanguticum and D. moldavica SSR information.

\begin{tabular}{lccccccccc}
\hline Species & Total & P1 & P2 & P3 & P4 & Pc & LSC & SSC & IR \\
\hline D. tanguticum & 51 & 32 & 5 & 1 & 2 & 11 & 44 & 3 & $2(2)$ \\
D. moldavica & 54 & 35 & 6 & 1 & 3 & 9 & 45 & 5 & $2(2)$ \\
\hline
\end{tabular}

P1: single-nucleotide; P2: double-nucleotide; P3: three-nucleotide; P4: fournucleotide; Pc: complex-nucleotide.

D. moldavica were 32 (62.75\%) and 35 (64.81\%), respectively, with a large proportion. Among SSRs of D. tanguticum and D. moldavica, the four-nucleotide repeat sequences are much more than three-nucleotide repeat sequences, which is consistent with the study of Salvia miltiorrhiza [38, 39].

Genes such as $r b c L$, $m a t K$, and $a t p B$ are widely applied as molecular markers in general phylogenetic studies [40]. After analysis of the SSRs of D. tanguticum and D. moldavica, we

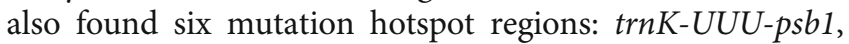
trnR-UCU-atpI, rpoC2-trnC-GCA, psaA-trnL-UAA, accDcemA, and $\operatorname{clp} P$. Five of them are located in the intergenic region (IGS), and one is located in the intron region. Through comparison of the two Dracocephalum plants, the order of mutation rate from high to low is the noncoding region, the intron region, the LSC region, the SSC region, and the IR region.

2.4. Evolutionary and Phylogenetic Analysis. In this study, we combined the sequenced chloroplast genomes of two Dracocephalum plants with that of 30 other angiosperms, constructed a phylogenetic tree with RAxML v8.2.10 (https:// cme.h-its.org/exelixis/software.html) software based on the maximum likelihood (ML) method, and selected Cistanche deserticola (NC_021111.1) as the outgroup. Phylogenetic trees based on complete chloroplast genome data have a higher bootstrap value (the branch with a value greater than 75 is considered as a more stable branch). The results showed that $D$. tanguticum and $D$. moldavica belong to the same genus. Although the D. tanguticum is more similar to Rosmarinus morphology, the phylogenetic tree shows that the relationship between the two Dracocephalum plants and Mentha is closer than that with Rosmarinus (Figure 4). This result is consistent with traditional taxonomy. In the seed morphology, Dracocephalum is closer to Mentha [28, 41].

\section{Materials and Methods}

3.1. Material Acquisition. We used the leaves of D. tanguticum and D. moldavica for chloroplast genome sequencing. The vigorous, healthy, and fresh leaves of $D$. tanguticum and D. moldavica were obtained from the Science and Technology Park of Xizang Agriculture and Animal Husbandry College, Linzhi City, Tibet Autonomous Region, China. All leaves were immediately frozen in liquid nitrogen and stored at $-80^{\circ} \mathrm{C}$ before analysis and use.

3.2. Sequencing and Assembly of Chloroplast Genomes. The modified CTAB (cetrimonium bromide) method was used to extract total genomic DNA from fresh leaves. Sheared low molecular weight DNA fragments were used to construct paired-end (PE) libraries according to the protocol of the Illumina manual (San Diego, CA, USA). Completed libraries were pooled and sequenced in the Illumina NovaSeq platform with PE150 sequencing strategy and $350 \mathrm{bp}$ insert size (Genepioneer Biotechnologies Co. Ltd., Nanjing, Jiangsu, China). We used the clean data to assemble the chloroplast 


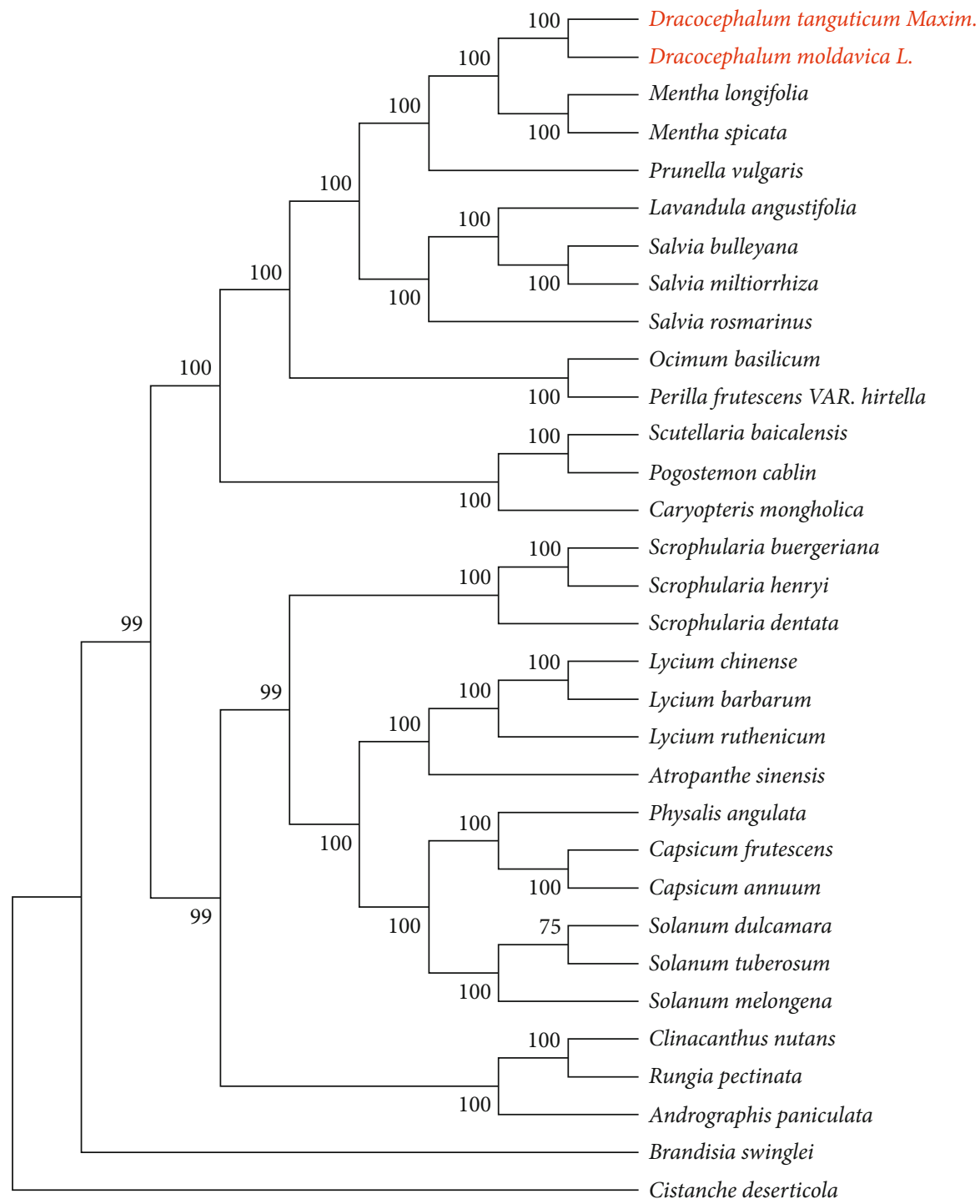

Figure 4: Phylogenetic tree constructed from the complete chloroplast genome.

genomes with SPAdes v3.10.1 (http://cab.spbu.ru/software/ spades/) software.

\subsection{Annotation and Analysis of Chloroplast Genomes.} CpGAVAS was used to annotate the sequence before manual correction. The blast v2.2.25 (https://blast.ncbi.nlm.nih.gov/ Blast.cgi) software was used to compare the chloroplast genome's cds sequence on NCBI to correct cds; the hmmer v3.1b2 (http://www.hmmer.org/) software was used to correct the rRNA sequence; the aragorn v1.2.38 (http://130.235 .244.92/ARAGORN/) software was used to correct the tRNA. The OGDRAW program (https://chlorobox.mpimp-golm .mpg.de/OGDraw. html) was used to make a circular map of the chloroplast genome. The base content was analyzed with biological editing software, and the nucleotide diversity (RSCU) was analyzed with MEGA 7 software.

We used REPuter (http://bibiserv.techfak.uni-bielefeld .de/ter/) to look for and analyze the size and position of forward, reverse, palindrome, and complementary repeat sequences. MISA (http://pgrc.ipk.gatersleben.de/misa/) was adopted to identify SSRs, the threshold for single nucleotide SSRs is 10 repeats, the threshold for double-nucleotide SSRs is 5 repeats, the threshold for three-nucleotide SSRs is 4 repeats, and the threshold of four-, five-, and six-nucleotide SSRs is 3 repeats. The mauve software was used to sequence alignment of the chloroplast genome.

3.4. Phylogenetic Analysis. Based on the chloroplast genome data of $D$. tanguticum and D. moldavica and the chloroplast genomes of 30 other species, a phylogenetic tree was constructed, and Cistanche deserticola (NC_021111.1) chloroplast genome was selected as the outgroup. Before constructing the phylogenetic tree, we used MAFFT software (V7.427, auto mode) for multisequence alignment to obtain neatly arranged chloroplast genomes for phylogenetic analysis. By default, the whole genome is used for evolutionary tree analysis, and the ring sequence is set at the same starting point. MAFFT software was used to perform multiple sequence alignment between species sequences, and tri$\mathrm{mAl}$ (v1.4.rev15) was used to prune the aligned data. Then, RAxML V8.2.10 (https://cme.h-its.org/exelixis/software.html) 
software was used, its GTRGAMMA model was selected for Bootstrap analysis, and the Bootstrap $=1000$ was set to build the maximum likelihood evolutionary tree.

\section{Conclusion}

The size, GC content, gene order, and number of chloroplast genomes in D. tanguticum and D. moldavica are highly similar. As D. moldavica lacks the rps2 gene compared to D. tanguticum, which may be one of the reasons that $D$. moldavica is $1,086 \mathrm{bp}$ shorter than D. tanguticum in the full length of the chloroplast genome. Through SSR analysis, six mutation hotspot regions were found, among which five were located in the intergenic region, and one was located in the intron region. Through phylogenetic tree analysis, it was found that Mentha and Dracocephalum have a closer genetic relationship. In this study, we have provided the complete chloroplast genome of $D$. tanguticum and D. moldavica, which provides a basis for the identification and overcoming of phylogenetic problems at the species level.

\section{Data Availability}

The complete chloroplast genome data used to support the findings of this study have been deposited in the NCBI repository (the accession numbers of $D$. tanguticum and D. moldavica are MT457746 and MT457747, respectively).

\section{Conflicts of Interest}

The authors declare that they have no known competing financial interests or personal relationships that could have appeared to influence the work reported in this paper.

\section{Authors' Contributions}

The experimental design was completed by J.Y., K.Z., and J.L.; acquisition and treatment of plant materials were completed by Y.X., Y.S., and P.H.; data processing and analysis were performed by J.Y., F.Z., H.Q., M.Y., B.X., and J.M.; the paper was written by J.Y. and Z.L.; X.L. put forward key opinions and revised the paper.

\section{Acknowledgments}

This work was financially supported by the National Natural Science Foundation of China (No. 81660628), Tibet Autonomous Region Major Special Science and Technology Project (No. XZ201901-GA-04), Budget Project of Tibet Autonomous Region Level (2019-44), the Forth National Survey of Traditional Chinese Medicine Resources, Chinese or Tibet Medicinal Resources Investigation in Tibet Autonomous Region (State Administration of Chinese Traditional Medicine 20200501-542330), Capacity Construction of National Traditional Chinese Medicine Inheritance and Innovation Project (cc-cstc-CA-19-2) and Key Project at Central Government Level: The Ability Establishment of Sustainable Use for Valuable Chinese Medicine Resources (Nos. 2060302-1704-09 and 2060302-1702-13), and Xizang Agriculture and Animal Husbandry College Graduate Innovation Program Fund (YJS2019-14).

\section{References}

[1] Lamiaceae plant familyhttp://global.britannica.com/plant/ lamiaceae, http://Britannica.com.

[2] J. Bunsawat, N. E. Elliott, K. L. Hertweck, E. Sproles, and L. A. Alice, "Phylogenetics of Mentha (Lamiaceae): evidence from chloroplast DNA sequences," Systematic Botany, vol. 29, no. 4, pp. 959-964, 2004.

[3] W. Li, J. Yin, B. Ye et al., "Deletion of a DnaK protein gene causes seedling green-revertible albino by retarding chloroplast development in rice," Science Bulletin, vol. 60, no. 23, pp. 2054-2058, 2015.

[4] S. Greiner, H. Golczyk, I. Malinova et al., "Chloroplast nucleoids are highly dynamic in ploidy, number, and structure during angiosperm leaf development," The Plant Journal, vol. 102, no. 4, pp. 730-746, 2020.

[5] S. Kim and Y. Suh, "Phylogeny of Magnoliaceae based on ten chloroplast DNA regions," Journal of Plant Biology, vol. 56, no. 5, pp. 290-305, 2013.

[6] P. Maliga, "Towards plastid transformation in flowering plants," Trends in Biotechnology, vol. 11, no. 3, pp. 101-107, 1993.

[7] T. Nonoyama, E. Kazamia, H. Nawaly et al., "Metabolic innovations underpinning the origin and diversification of the diatom chloroplast," Biomolecules, vol. 9, no. 8, p. 322, 2019.

[8] J. Palmer, "Comparative organization of chloroplast genomes," Annual Review of Genetics, vol. 19, no. 1, pp. 325-354, 1985.

[9] J. Carbonell-Caballero, R. Alonso, V. Ibañez, J. Terol, M. Talon, and J. Dopazo, “A phylogenetic analysis of 34 chloroplast genomes elucidates the relationships between wild and domestic species within the genus citrus," Molecular Biology and Evolution, vol. 32, no. 8, pp. 2015-2035, 2015.

[10] P. K. Ingvarsson, S. Ribstein, and D. R. Taylor, "Molecular evolution of insertions and deletion in the chloroplast genome of Silene," Molecular Biology and Evolution, vol. 20, no. 11, pp. 1737-1740, 2003.

[11] T. W. Chumley, J. D. Palmer, J. P. Mower et al., "The complete chloroplast genome sequence of Pelargonium $\times$ hortorum: Organization and evolution of the largest and most highly rearranged chloroplast genome of land plants," Molecular Biology and Evolution, vol. 23, no. 11, pp. 2175-2190, 2006.

[12] T. Wakasugi, J. Tsudzuki, S. Ito, T. Tsudzuki, M. Sugiura, and M. Sugiura, "Loss of all ndh genes as determined by sequencing the entire chloroplast genome of the black pine Pinus thunbergii," Proceedings of the National Academy of Sciences, vol. 91, no. 21, pp. 9794-9798, 1994.

[13] J. Chen, Z. Hao, H. Xu et al., "The complete chloroplast genome sequence of the relict woody plant Metasequoia glyptostroboides Hu et Cheng," Frontiers in Plant Science, vol. 6, p. 447, 2015.

[14] X. Li, Y. Li, M. Zang, M. Li, and Y. Fang, "Complete chloroplast genome sequence and phylogenetic analysis of Quercus acutissima," International Journal of Molecular Sciences, vol. 19, no. 8, p. 2443, 2018.

[15] S. Xue, T. Shi, W. Luo et al., "Comparative analysis of the complete chloroplast genome among Prunus mume, P. armeniaca, and P. salicina," Horticulture Research, vol. 6, no. 1, 2019.

[16] D. Jiang, Z. Zhao, T. Zhang et al., "The chloroplast genome sequence of Scutellaria baicalensis provides insight into intraspecific and interspecific chloroplast genome diversity in Scutellaria," Genes, vol. 8, no. 9, p. 227, 2017. 
[17] C. C. Lai, P. H. Huang, A. H. Yang et al., "Baicalein attenuates lung injury induced by myocardial ischemia and reperfusion," The American Journal of Chinese Medicine, vol. 45, no. 4, pp. 791-811, 2017.

[18] T. Borsch and D. Quandt, "Mutational dynamics and phylogenetic utility of noncoding chloroplast DNA," Plant Systematics and Evolution, vol. 282, no. 3-4, pp. 169-199, 2009.

[19] K. Shinozaki, M. Ohme, M. Tanaka et al., "The complete nucleotide sequence of the tobacco chloroplast genome: its gene organization and expression," The EMBO Journal, vol. 5, no. 9, pp. 2043-2049, 1986.

[20] W. Dong, T. Cheng, C. Li et al., "Discriminating plants using the DNA barcoderbcLb: an appraisal based on a large data set," Molecular Ecology Resources, vol. 14, no. 2, pp. 336-343, 2014.

[21] P. B. Pelser, A. H. Kennedy, E. J. Tepe et al., "Patterns and causes of incongruence between plastid and nuclear Senecioneae (Asteraceae) phylogenies," American Journal of Botany, vol. 97, no. 5, pp. 856-873, 2010.

[22] O. A. Pérez-Escobar, J. A. Balbuena, and M. Gottschling, "Gottschling. Rumbling orchids: how to assess divergent evolution between chloroplast endosymbionts and the nuclear host," vol. 65, no. 1, pp. 51-65, 2016.

[23] Y. He, H. Xiao, C. Deng, L. Xiong, J. Yang, and C. Peng, “The complete chloroplast genome sequences of the medicinal plant Pogostemon cablin," International Journal of Molecular Sciences, vol. 17, no. 6, p. 820, 2016.

[24] J.-B. Yang, S.-X. Yang, H.-T. Li, J. Yang, and D.-Z. Li, "Comparative chloroplast genomes of Camellia species," Plos One, vol. 8, no. 8, article e73053, 2013.

[25] H. Daniell, K. J. Wurdack, A. Kanagaraj, S. B. Lee, C. Saski, and R. K. Jansen, "The complete nucleotide sequence of the cassava (Manihot esculenta) chloroplast genome and the evolution of atpF in Malpighiales: RNA editing and multiple losses of a group II intron," Theoretical and Applied Genetics, vol. 116, no. 5, pp. 723-737, 2008.

[26] C. Saski, S. B. Lee, H. Daniell et al., "Complete chloroplast genome sequence of Gycine max and comparative analyses with other legume genomes," Plant Molecular Biology, vol. 59, no. 2, pp. 309-322, 2005.

[27] K. Kim, S. C. Lee, J. Lee et al., "Comprehensive survey of genetic diversity in chloroplast genomes and $45 \mathrm{~S}$ nrDNAs within Panax ginseng species," PLoS One, vol. 10, no. 6, article e0117159, 2015.

[28] S. Liqun, Comparison and Evolutionary Analysis of the Whole Genome and Family of Chloroplast of Three Medicinal Plants from Labiaceae, Zhejiang University, 2018.

[29] K. Wang, L. Li, Y. Hua et al., "The complete chloroplast genome ofMentha spicata,an endangered species native to South Europe," Mitochondrial DNA Part B, vol. 2, no. 2, pp. 907-909, 2017.

[30] L. Sun, Z. Jiang, X. Wan et al., "The complete chloroplast genome of_Magnolia polytepala_: Comparative analyses offer implication for genetics and phylogeny of Yulania," Gene, vol. 736, p. 144410, 2020.

[31] H. Cheng, J. Li, H. Zhang et al., "The complete chloroplast genome sequence of strawberry (Fragaria $\mathrm{x}$ ananassa Duch.) and comparison with related species of Rosaceae," Peer J, vol. 5, article e3919, 2017.

[32] L. Ni, Z. Zhao, G. Dorje, and M. Ma, "The complete chloroplast genome of YeXing-Ba (Scrophularia dentata; Scrophulariaceae), an alpine Tibetan herb," PLoS One, vol. 11, no. 7, article e0158488, 2016.
[33] S. H. Kazakoff, M. Imelfort, D. Edwards et al., "Capturing the biofuel wellhead and powerhouse: the chloroplast and mitochondrial genomes of the leguminous feedstock tree Pongamia pinnata," PLoS One, vol. 7, no. 12, article e51687, 2012.

[34] V. Ravi, J. P. Khurana, A. K. Tyagi, and P. Khurana, "An update on chloroplast genomes," Plant Systematics and Evolution, vol. 271, pp. 101-122, 2007.

[35] S. E. Goulding, R. G. Olmstead, C. W. Morden, and K. H. Wolfe, "Ebb and flow of the chloroplast inverted repeat," Molecular \& General Genetics, vol. 252, no. 1-2, pp. 195-206, 1996.

[36] Q.-p. Zhang, X. Wei, W.-s. Liu et al., "The genetic relationship and structure of some natural interspecific hybrids in Prunus subgenus Prunophora, based on nuclear and chloroplast simple sequence repeats," Genetic Resources and Crop Evolution, vol. 65, pp. 625-636, 2018.

[37] S. Kurtz, J. V. Choudhuri, E. Ohlebusch, C. Schleiermacher, J. Stoye, and R. Giegerich, "REPuter: the manifold applications of repeat analysis on a genomic scale," Nucleic Acids Research, vol. 29, no. 22, pp. 4633-4642, 2001.

[38] J. Qian, J. Song, H. Gao et al., "The complete chloroplast genome sequence of the medicinal plant Salvia miltiorrhiza," PLoS One, vol. 8, no. 2, article e57607, 2013.

[39] C. Liang, L. Wang, J. Lei et al., "A Comparative Analysis of the Chloroplast Genomes of Four Salvia Medicinal Plants," Engineering, vol. 5, no. 5, pp. 907-915, 2019.

[40] H. Katayama and C. Uematsu, "Structural analysis of chloroplast DNA in Prunus (Rosaceae): evolution, genetic diversity and unequal mutations," Theoretical and Applied Genetics, vol. 111, no. 7, pp. 1430-1439, 2005.

[41] Z. Wu and X. Li, "On the evolution and distribution in Labiatae," Acta Botanica Yunnanica, vol. 2, pp. 97-118, 1982. 